\title{
COMMENTARY
}

\section{Comparison of Illumina paired-end and single-direction sequencing for microbial 16S rRNA gene amplicon surveys}

\author{
Jeffrey J Werner, Dennis Zhou, J Gregory Caporaso, Rob Knight and Largus T Angenent
}

The ISME Journal (2012) 6, 1273-1276; doi:10.1038/ ismej.2011.186; published online 15 December 2011

High-throughput sequencing of $16 \mathrm{~S}$ rRNA gene amplicons is a valuable tool for comparing microbial community structure among hundreds of samples for which researchers have primarily used the 454 Pyrosequencing platform. The Illumina platform produces far more reads than 454 (up to 1.5 billion reads per run, compared with 1 million reads per run on a 454 plate of comparable cost), but produces fewer base pairs (bp) per read (75-150 bp per read compared with $250-400$ bp per read on 454). The shorter Illumina reads may reduce phylogenetic resolution, both in terms of picking operational taxonomic units (OTUs) and determining evolutionary distances between OTUs. The paired-end (PE) approach where each molecule is sequenced from both the $5^{\prime}$ and $3^{\prime}$ ends can double the number of bp per read for the Illumina platform. Some researchers have obtained overlapping PE Illumina reads covering the V3 (Bartram et al., 2011) or V6 (Zhou et al., 2011) regions of $16 \mathrm{~S}$ rRNA genes, but many other useful primer regions (for example, V1-V2 or V4) are $>200 \mathrm{bp}$ in length in which case $\mathrm{PE}$ reads do not overlap. However, it is possible, as we present here, to use non-overlapping $\mathrm{PE}$ reads to pick OTUs and build a phylogenetic tree. We assessed the utility of using PE Illumina sequencing, compared with results from single-direction (SD) sequencing from the $5^{\prime}$ position of the V4 region of $16 \mathrm{~S}$ rRNA genes. We compared alpha- and beta-diversity analyses (species richness and between-sample comparisons, respectively), using previously published non-overlapping PE Illumina sequence data from $16 \mathrm{~S}$ rRNA gene surveys of 28 human microbiome and environmental samples (Caporaso et al., 2011).

Illumina sequences were quality filtered using the default pipeline in QIIME 1.2.1 (Caporaso et al., 2010b), including the default quality thresholds and a minimum read length of $75 \mathrm{bp}$. To avoid potential biases from quality filtering, only sequences passing the quality threshold in both $5^{\prime}$ and $3^{\prime}$ reads were kept for downstream processing $(43 \%$ of the total reads were removed because one of the two directions did not pass the quality threshold). All reads were trimmed to $75 \mathrm{bp}$ length (total of $150 \mathrm{bp}$ per seq for $\mathrm{PE}$ ). The $\mathrm{PE}$ and $5^{\prime} \mathrm{SD}$ data were analyzed separately, using the default settings in QIIME, with the following additional steps for PE data: before OTU-picking with uclust (97\% ID) (Edgar, 2010), we joined $\mathrm{PE}$ reads 'inside-out' such that the $3^{\prime}$ end of the $3^{\prime}$ read was to the left of the $5^{\prime}$ end of the $5^{\prime}$ read, required for uclust to perform accurate pairwise alignments. Based on a simulation using Greengenes sequences trimmed to the V4 region, we found that uclust assigned similar pairwise alignment distances to inside-out reads compared with the normal configuration (Supplementary Figure S1). OTU representative sequences (separate $3^{\prime}$ and $5^{\prime}$ reads for PE) were aligned with PyNAST (Caporaso et al., 2010a) against separate regions of the August 2007 Greengenes core (DeSantis et al., 2006), trimmed to positions 2250-2423 for $5^{\prime}$ reads and 3805-4069 for $3^{\prime}$ reads. Trimming reference sequences had advantages of rapid computation, more stringent discarding of non-16S reads, and has previously been shown to improve taxonomic classification (Werner et al., 2011b). We built a phylogenetic tree (FastTree) (Price et al., 2010) from aligned, filtered sequences (for PE, the separate $5^{\prime}$ and $3^{\prime}$ alignments were joined end-to-end to use all $150 \mathrm{bp}$ for phylogeny). Separately for each method, we discarded OTUs with fewer than 10 total reads $2.6 \%$ of the 16.6 million high-quality reads). This was done to discard false diversity because of sequencing errors, by setting a $10 \times$ threshold of evidence needed to support a true sequence. OTUs that failed to align $>72 \mathrm{bp}(0.007 \%$ of remaining reads) were also removed.

We obtained slightly higher alpha-diversity (Chao1) using PE data compared with 5' SD data, but the results were still comparable (Supplementary Figure S2). Between-sample comparisons of alpha-diversity were consistent, except for the fecal samples, which had greater disagreement. We also observed that the pairing of sequences into the same OTU was least consistent for fecal and soil samples, comparing PE and 5' SD data (Supplementary Figure S3). We additionally used three beta-diversity metrics to calculate distances between samples in both the PE data and the $5^{\prime}$ SD read data: BrayCurtis (based on relative abundances of OTUs), 
unweighted UniFrac (based on phylogenetic structure), and weighted UniFrac (based on phylogenetic structure, weighted by OTU abundances). All three metrics have a scale from 0 to 1 .

Bray-Curtis distances for OTUs picked based on $P E$ reads vs $5^{\prime}$ SD reads were closely correlated (Supplementary Figure S4A; $R^{2}=0.993$ ). Disagreement between absolute distances was below 0.1 (Supplementary Figure S4D), and the UPGMA clustering of samples was comparable between methods (Supplementary Figure S5). Thus, the two OTU-picking strategies resulted in the same betadiversity results when measured using a distance metric based solely on OTU picking.

We used the UniFrac distance metric (Lozupone and Knight, 2005) to compare sample clustering based on phylogenetic structure. Unweighted UniFrac has provided useful results in a number of microbiome studies (Ley et al., 2008; Costello et al., 2009; Werner et al., 2011a). There were no practical differences between unweighted UniFrac clustering results from PE data (Figure 1a) and 5' SD data (Figure 1b).
Distances were closely correlated between methods (Supplementary Figure S4B; $R^{2}=0.982$ ) and absolute differences were below 0.1 (Supplementary Figure S4E). However, abundance-weighted UniFrac produced different results between the two methods (Supplementary Figure S4C and F; $R^{2}=0.811$; absolute differences up to 0.3), which resulted in more consistent sample clustering from the $\mathrm{PE}$ data compared with $5^{\prime}$ SD data (Figure 2). These patterns were verified using ordination and Procrustes analysis (Supplementary Figure S6).

On the basis of these results, we expect that future 16S rRNA gene surveys using SD reads in the V4 region will yield similar OTU profiles and unweighted UniFrac results, compared with the significantly more expensive $\mathrm{PE}$ approach. However, some research questions may require the weighted UniFrac metric, and the non-overlapping $\mathrm{PE}$ approach presented here yielded moderate improvements in sample-weighted UniFrac clustering. Also important to note, and not considered here, are the advantages of overlapping PE reads, a Unweighted UniFrac using paired-end reads

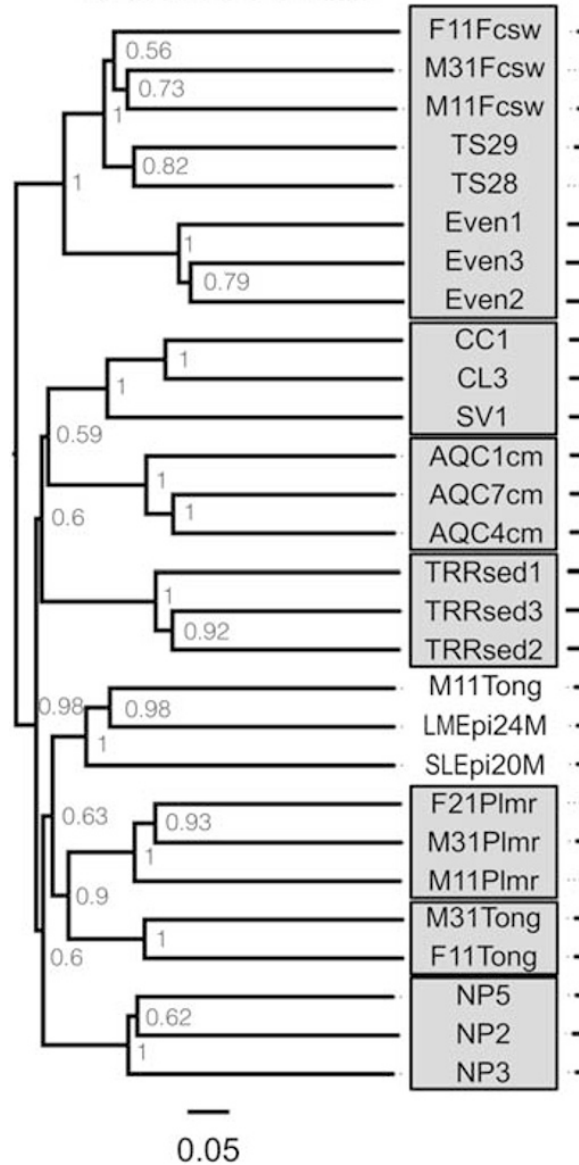

b Unweighted UniFrac Using 5' single-direction reads

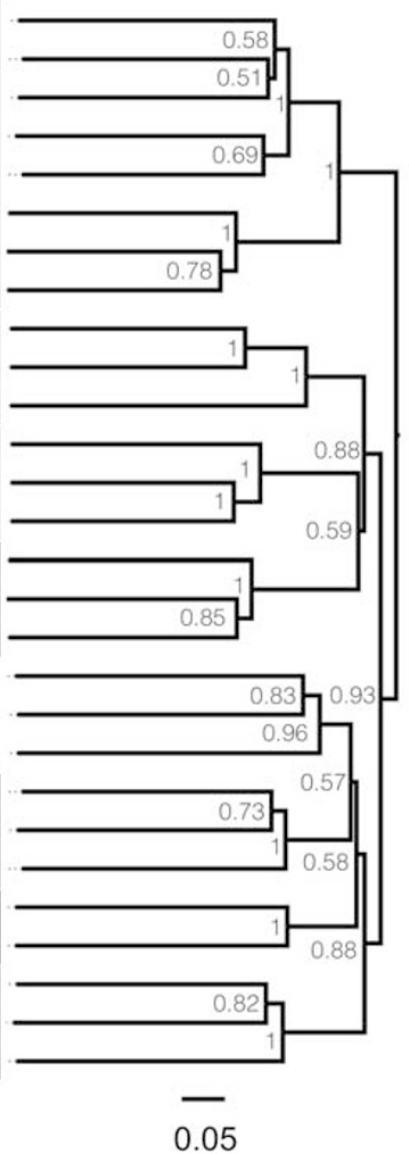

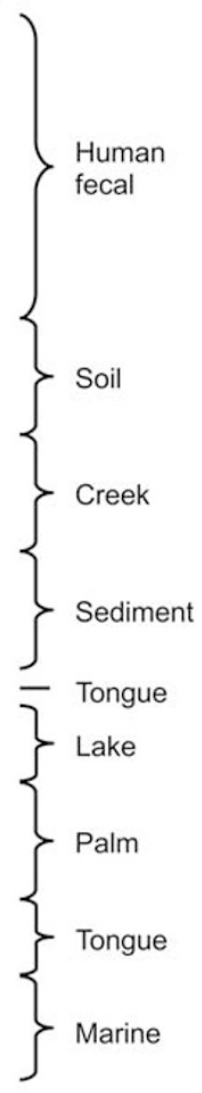

0.05

Figure 1 Choice of PE or SD reads made no practical difference in phylogenetic clustering of samples: UPGMA tree of unweighted UniFrac distances between samples determined using the PE sequence data (a) as well as SD reads (b). Bootstrap values represent 100 rarefactions of 50000 sequences per sample. 
a

Weighted UniFrac using paired-end reads

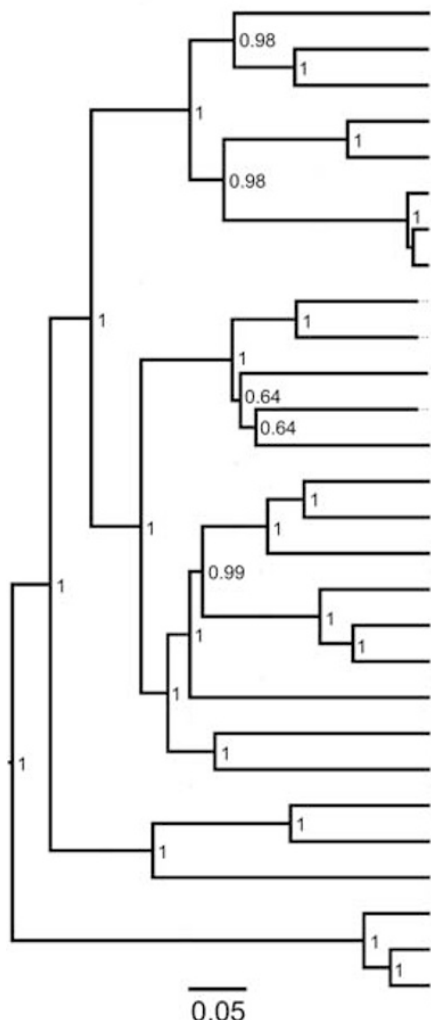

b Weighted UniFrac using 5 ' single-direction reads

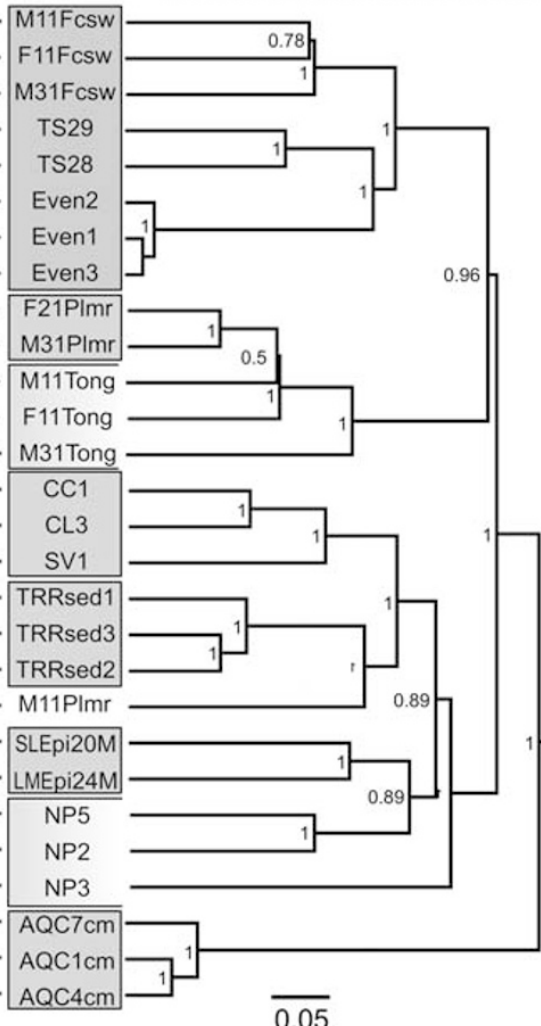

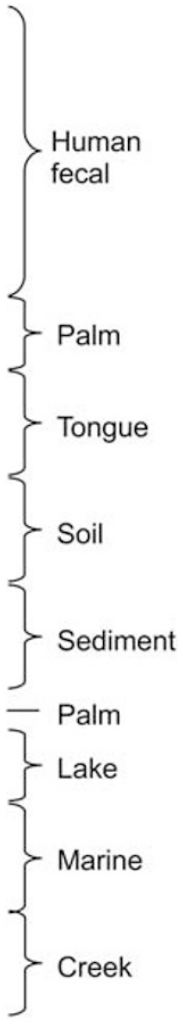

Figure 2 Choice of PE or SD reads affected the higher-order branching of abundance-weighted UniFrac clustering of samples: UPGMA tree of weighted UniFrac distances between samples determined using the PE sequence data (a) as well as SD reads (b). Bootstrap values represent 100 rarefactions of 50000 sequences per sample. Note the more consistent clustering of marine and tongue samples using PE reads.

including error correction, though this is currently not possible in the $\mathrm{V} 4$ region.

\section{Acknowledgements}

This work was funded by the USDA through the National Institute of Food and Agriculture (NIFA), grant number 2007-35504-05381 to LTA, the Cornell University Agricultural Experiment Station federal formula funds NYC-123444 received from the USDA NIFA to LTA, and the Howard Hughes Medical Institute to RK.

\section{JJ Werner is at Chemistry Department, SUNY} Cortland, Cortland, NY, USA;

$J J$ Werner is at Department of Biological and Environmental Engineering, Cornell University, Ithaca, NY, USA;

$D$ Zhou is at Department of Biological and Environmental Engineering, Cornell University, Ithaca, NY, USA; JG Caporaso is at Department of Computer Science, Northern Arizona University, Flagstaff, AZ, USA;

$R$ Knight is at Department of Chemistry and Biochemistry, University of Colorado at Boulder, Boulder, CO, USA and
LT Angenent is at Department of Biological and Environmental Engineering, Cornell University,

Ithaca, NY, USA

E-mail: la249@Cornell.edu

\section{References}

Bartram AK, Lynch MDJ, Stearns JC, Moreno-Hagelsieb G, Neufeld JD. (2011). Generation of multimillionsequence 16S rRNA gene libraries from complex microbial communities by assembling paired-end Illumina reads. Appl Environ Microbiol 77: 3846-3852.

Caporaso JG, Bittinger K, Bushman FD, DeSantis TZ, Andersen GL, Knight R. (2010a). PyNAST: a flexible tool for aligning sequences to a template alignment. Bioinformatics 26: 266-267.

Caporaso JG, Kuczynski J, Stombaugh J, Bittinger K, Bushman FD, Costello EK et al. (2010b). QIIME allows analysis of high-throughput community sequencing data. Nat Meth 7: 335-336.

Caporaso JG, Lauber CL, Walters WA, Berg-Lyons D, Lozupone C, Turnbaugh PJ et al. (2011). Global patterns of 16S rRNA diversity at a depth of millions of sequences per sample. Proc Natl Acad Sci USA 108: 4516-4522.

Costello EK, Lauber CL, Hamady M, Fierer N, Gordon JI, Knight R. (2009). Bacterial community variation in 
human body habitats across space and time. Science 326: 1694-1697.

DeSantis TZ, Hugenholtz P, Larsen N, Rojas M, Brodie EL, Keller K et al. (2006). Greengenes, a chimera-checked $16 \mathrm{~S}$ rRNA gene database and workbench compatible with ARB. Appl Environ Microbiol 72: 5069-5072.

Edgar RC. (2010). Search and clustering orders of magnitude faster than BLAST. Bioinformatics 26: $2460-2461$.

Ley RE, Hamady M, Lozupone C, Turnbaugh PJ, Ramey RR, Bircher JS et al. (2008). Evolution of mammals and their gut microbes. Science 320: 1647-1651.

Lozupone C, Knight R. (2005). UniFrac: a new phylogenetic method for comparing microbial communities. Appl Environ Microbiol 71: 8228-8235.
Price MN, Dehal PS, Arkin AP. (2010). FastTree 2-approximately maximum-likelihood trees for large alignments. Plos One 5: e9490.

Werner JJ, Knights D, Garcia ML, Scalfone NB, Smith S, Yarasheski K et al. (2011a). Bacterial community structures are unique and resilient in full-scale bioenergy systems. Proc Natl Acad Sci USA 108: 4158-4163.

Werner JJ, Koren O, Hugenholtz P, DeSantis TZ, Walters WA, Caporaso JG et al. (2011b). Impact of training sets on classification of high-throughput bacterial 16S rRNA gene surveys. ISME J; e-pub ahead of print 30 June 2011; doi:10.1038/ismej.2011.82.

Zhou HW, Li DF, Tam NFY, Jiang XT, Zhang H, Sheng HF et al. (2011). BIPES, a cost-effective high-throughput method for assessing microbial diversity. ISME J 5: 741-749.

Supplementary Information accompanies the paper on The ISME Journal website (http://www.nature.com/ismej) 